Rev. Psicol. (Arequipa. Univ. Catól. San Pablo) / Año 2020 / Vol 10 / № 2 / pp. 27-35

ISSN 2306-0565 versión impresa / ISSN 2311-7397 versión on line

(C) $\$ \bigoplus \begin{aligned} & \text { Esta obra está bajo una Licencia Creative Commons } \\ & \text { Atribución 4.o Internacional (BY-NC-ND) }\end{aligned}$

\title{
COMPARACIÓN DE LA TOMA DE DECISIONES \\ Y EL FUNCIONAMIENTO EJECUTIVO EN FUNCIÓN \\ DE LA RELIGIÓN EN UNIVERSITARIOS DE AREQUIPA
}

\section{COMPARISON OF DECISION-MAKING AND EXECUTIVE FUNCTIONING \\ ACCORDING TO RELIGION AMONG UNIVERSITY STUDENTS IN AREQUIPA}

\author{
Claudia Angulo Calle $e^{1}$ Adriana Aragón Velazco ${ }^{1}$, María Chávez Perea ${ }^{1}$ \\ María Escobar ${ }^{2}$ y Rafaela Molina Romero ${ }^{1}$ \\ 1. Universidad Católica San Pablo, Arequipa, Perú \\ 2. Universidad Nacional de Santiago del Estero, Argentina
}

\begin{abstract}
Resumen
Uno de los factores influyentes en la toma de decisiones y el comportamiento de una persona, es la religión. El objetivo de esta investigación es valorar si existen diferencias en la toma de decisiones y el funcionamiento ejecutivo tomando como criterio de comparación, la religión. Para ello, se tomó una muestra conformada por estudiantes de una universidad privada de Arequipa, a quienes se les aplicó el IOWA Gambling Task, el Eriksen Flanker Task y un formulario de creencias. Siendo así, en los resultados no se observaron diferencias significativas en base al criterio de religión sobre las variables de toma de decisiones y funcionamiento ejecutivo.
\end{abstract}

Palabras clave: Religión, funciones ejecutivas, toma de decisiones.

\begin{abstract}
One of the influencing factors in decision-making and a person's behavior is religion. The objective of this research is to assess whether there are differences in decision-making and executive functioning, taking religion as a criterion for comparison. To do this, a sample of students from a private university in
\end{abstract}




\begin{abstract}
Arequipa was recruited to whom the IOWA Gambling Task, the Eriksen Flanker Task and a belief form were administered. The results indicated there were no significant differences based on the criterion of religion for the variables of decision-making and executive functioning.
\end{abstract}

Key words: Religion, executive functioning, decision-making.

\section{Introducción}

Con el paso de los años, la influencia de la religión en la toma de decisiones de las personas se ha visto afectada por diversas razones. Una de ellas es la idea que la religión es un límite para la libertad de cada persona. Adicionalmente se argumenta que la adopción de un dogma no se da conscientemente, y por ende, en ausencia de un consentimiento; pues normalmente es desde la primera infancia que se enseña acerca de ello, por lo tanto, el niño no hace uso pleno de su plena voluntad y es ciertamente alentado a seguir determinada religión.

Tal conformidad en cuanto a imposiciones va tornándose en un pensamiento más individual y diferenciado en la adolescencia. Dentro de ésta, podemos distinguir dos etapas: la adolescencia temprana, que va desde los 11 a los 13 años, y la adolescencia tardía, de los 14 a los 20 . No es entonces, hasta en la segunda etapa que se alcanza un grado de maduración cerebral que permite el uso de los lóbulos frontales para la toma de decisiones; a diferencia de los adolescentes tempranos, que hacen uso de la amígdala, llevándolos a reacciones más emocionales e instintivas. Al llegar a esta etapa de maduración, la toma de decisiones se vuelve más racional, y a su vez, las conexiones neuronales se fortalecen y homogenizan, lo que incrementa la eficiencia del procesamiento cognitivo (Papalia, Wendkos y Duskin, 2009).

En cuanto al abordaje psicológico del funcionamiento ejecutivo y toma de decisiones, algunos autores proponen las siguientes afirmaciones.

Las funciones ejecutivas constituyen un controvertido constructo, bajo el cual se han agrupado diferentes procesos asociados al control consciente del pensamiento, comportamiento y afectividad. Entre los mismos pueden ser mencionados: la memoria de trabajo, el control atencional, el control inhibitorio, la toma de decisiones, entre otros. La capacidad de toma de decisiones, ha sido caracterizada como la habilidad para elegir entre alternativas vinculadas a determinados montos desconocidos de recompensa y castigo. (Bechara, Damasio \& Damasio, 200o, p. 181)

Por tal motivo, es de suma importancia destacar el papel de la toma de decisiones en adolescentes maduros y cómo es que ciertos factores, en este caso las creencias, influencian en este proceso.

Actualmente la religión continúa siendo un factor relevante en la sociedad peruana. Hecho que es evidente por medio de los 
datos obtenidos en el censo del 2007 a cargo del Instituto Nacional de Estadística e Informática (INEI), en donde se refleja que un $98.1 \%$ de la población peruana, se identifica con una religión y el $85.3 \%$ específicamente con la religión católica, quedando así un $1.9 \%$ perteneciente a la población atea y agnóstica (INEI, 2008).

Baumeister, Bauer y Lloyd (2010), plantean que la religión y los códigos morales han sido reconocidos como poderosos elementos en el control de la impulsividad, pues las creencias y emociones se ven reguladas por un mecanismo de control, que permite una mejor toma de decisiones, y paralelamente promueve un bienestar psicológico, facilitando una vida en sociedad. En pocas palabras, se hace referencia a la moralidad del mencionado mecanismo de control o en otros términos, el autocontrol.

Este mecanismo «permite a las personas superar sus propios deseos e impulsos para hacer lo mejor en orden a los intereses y estándares de la cultura» (Baumeister, \& Exline, 1999, p. 1166). La interrogante hace referencia entonces al discernimiento entre lo apropiado y lo no apropiado; cuestión que puede ser resuelta a la luz de la virtud, que es la encargada de realizar actos socialmente deseados y aceptados, a través de la asimilación de las reglas morales. Por logeneral, se alega que tales reglas son adaptaciones específicamente diseñadas para permitir a las personas vivir en armonía (Zegarra, \& Chino, 2016). Empero, en muchas ocasiones, tal como lo dice Baumeister y Exline (1999), la moral consiste o se basa en la obediencia de los designios divinos, por lo cual se cree que las personas religiosas tienden a desarrollar un mejor manejo de su impulsividad, producto de un análisis minucioso previo a la toma de decisiones.

Stuss y Benson (citados en TirapuUstárroz, García, Ríos-Lagos y Ardila, 2012) diseñaron una pirámide acerca de las funciones ejecutivas, en cuyo vértice hallamos la autoconciencia o autoanálisis, que se plasma en cómo actúa la relación entre las experiencias subjetivas actuales y las previas en la toma de decisiones, para resolver nuevos problemas y guiar la toma de decisiones para el futuro. La autoconciencia, depende de los inputs que recibe de los sistemas sensorial-perceptual y de control ejecutivo, mientras que su output influye en la naturaleza y el grado del control ejecutivo.

Por tales motivos es que el objetivo de esta investigación es conocer si existe una relación significativa entre la influencia de una creencia religiosa y la toma de decisiones y el funcionamiento ejecutivo en adolescentes maduros, y si es factible que este tipo de educación pueda fomentar una mejora en estos procesos.

\section{Método}

El estudio fue de tipo ex post facto retrospectivo simple, puesto que no se da una manipulación de las variables. Además, es un estudio transversal debido a que la evaluación se realizó en un solo momento y no en varios. Es también descriptivo comparativo porque describimos las características de nuestra muestra y 
realizamos la comparación de nuestras variables de estudio en base a la variable de criterio que es la religión (Ato, López, \& Benavente, 2013).

\section{Muestra}

Para este estudio se identificaron 82 estudiantes de diferentes escuelas profesionales de una universidad privada de la ciudad de Arequipa, quienes fueron seleccionados por métodos no probabilísticos, mediante la técnica de sujetos voluntarios (Hernández, Fernández, \& Baptista, 2006). Se evaluaron alumnos de ambos sexos con un rango de edad de 17 a 20 años respectivamente. En los creyentes de la religión católica la edad media fue de $18.86(\mathrm{DE}= \pm 1.50$; Rango $=16-20)$, en los creyentes de la fue religión cristiana la edad media fue de $19.13(\mathrm{DE}=$ \pm 1.12 ; Rango $=17-20$ ), en los creyentes de otras religiones la edad media fue de 19.50 $(\mathrm{DE}= \pm 0.71 ;$ Rango $=19-20)$ y en los no creyentes la edad media fue de 19.14 $(\mathrm{DE}= \pm 1.17 ;$ Rango $=17-20)$. Los sujetos se encontraban cursando entre el primer y séptimo semestre de estudios.

El porcentaje de participantes según el género en los creyentes de la religión católica fue $39,7 \%$ para los varones y de $60,3 \%$ para las mujeres; en los creyentes de la religión cristiana hubo $25,0 \%$ de varones y 75,o \% de mujeres), en otras religiones hubo 50,0\% de varones y 50,0\% de mujeres, y entre los no creyentes hubo $42,9 \%$ de varones y $57,1 \%$ de mujeres.

Tabla 1. Características de la Muestra

\begin{tabular}{|lccccc|}
\hline \multirow{2}{*}{ Edad } & & $\begin{array}{c}\text { Católico } \\
(\mathbf{n}=\mathbf{5} 8)\end{array}$ & $\begin{array}{c}\text { Cristiano } \\
(\mathbf{n}=\mathbf{8})\end{array}$ & $\begin{array}{c}\text { Otros } \\
(\mathbf{n}=\mathbf{2})\end{array}$ & $\begin{array}{c}\text { Ninguno } \\
(\mathbf{n}=\mathbf{1 4})\end{array}$ \\
\hline \multirow{2}{*}{ Género } & Masculino & $38.86 ; \pm 1.50$ & $19.13 ; \pm 1.12$ & $19.50 ; \pm 0.71$ & $19.14 ; \pm 1.17$ \\
\hline \multirow{3}{*}{ Semestre } & Femenino & $60.3 \%$ & $75.0 \%$ & $50.0 \%$ & $57.1 \%$ \\
& $\mathbf{1}$ & $10.3 \%$ & $12.5 \%$ & -- & $21.4 \%$ \\
& $\mathbf{2}$ & $6.9 \%$ & $12.5 \%$ & -- & $21.4 \%$ \\
& $\mathbf{3}$ & $36.2 \%$ & $62.5 \%$ & $50.0 \%$ & $28.6 \%$ \\
& $\mathbf{4}$ & $15.5 \%$ & $12.5 \%$ & -- & $7.1 \%$ \\
& $\mathbf{5}$ & $22.4 \%$ & -- & $50.0 \%$ & $21.4 \%$ \\
& $\mathbf{6}$ & $6.9 \%$ & -- & -- & -- \\
\hline \multirow{3}{*}{ Practicante } & 7 & $1.7 \%$ & -- & -- & -- \\
\hline & Sí & $56.9 \%$ & $75.0 \%$ & $50.0 \%$ & -- \\
& No & $43.1 \%$ & $25.0 \%$ & $50.0 \%$ & $100.0 \%$ \\
\hline
\end{tabular}




\section{Instrumentos}

Para la clasificación de los participantes, se utilizó un cuestionario con cinco criterios, edad, género, semestre, religión y práctica religiosa.

Asimismo, se utilizó el software PEBL 0.13 versión portable, la cual contiene una amplia gama de batería de pruebas estandarizadas, permitiendo obtener los datos eficaz y eficientemente.

Para evaluar la toma de decisiones se utilizó el IOWA Gambling Task (Bechara, Damasio, Tranel, \& Damasio, 2005), que consta de una baraja de cartas para valorar el riesgo-beneficio a través de las elecciones que hacen los evaluados según las indicaciones de los evaluadores. Después de 100 intentos se obtiene la cantidad de aciertos y fallos de los evaluados, a través de los marcadores somáticos (Bechara, Damasio, Damasio, \& Anderson, 1994) y una curva del aprendizaje emocional. Así, la finalidad de la prueba es que los evaluados obtengan la mejor recompensa posible (Gordillo, Arana, Salvador, \& Hernández, 2011).

Para la evaluación del funcionamiento ejecutivo se aplicó el Eriksen Flanker Task, que es un conjunto de pruebas de inhibición de respuestas que se usa para evaluar la capacidad de suprimir respuestas inapropiadas en un contexto particular (Eriksen, \& Eriksen, 1964). Ha sido ampliamente utilizado por psicólogos cognitivos que han encontrado en su efecto, una valiosa plataforma para estudios de atención, detección, distracción, control ejecutivo y otros aspectos de la cognición (Tiplady, Degia, \& Dickson 2005). La prueba se compone de una serie de flechas que se orientan hacia la izquierda o derecha, en donde el participante solamente debe tomar en cuenta la flecha que se ubica en el centro de la serie y de esta manera inhibir los estímulos laterales y distractores con dos teclas, Shift izquierdo o derecho, en el menor tiempo posible.

\section{Procedimiento}

Para la aplicación de los instrumentos, se solicitaron los permisos correspondientes ante las autoridades de una universidad privada, de donde provienen los participantes. Todos los estudiantes fueron evaluados dentro del horario de clases con la venia de los profesores encargados, y se les solicitó el consentimiento informado luego de explicárseles los fines de la investigación. Solo se evaluaron los estudiantes que desearon participar del estudio a solicitud de las autoras.

\section{Análisis estadístico}

Se analizaron las características de la muestra por medio de estadísticos de frecuencia y descriptivos. Asimismo, por medio de la prueba ANOVA se hizo un análisis de las diferencias de las medias y los puntajes de las variables evaluadas en base a nuestra variable de criterio de religión. A partir de la prueba de homogeneidad de Levene, se llevó a cabo un análisis post hoc con la prueba de Tukey para poder analizar la diferencia y diferenciación entre grupos. 


\section{Resultados}

Se hizo un análisis de ANOVA para estudiar diferencias significativas de las variables toma de decisiones y funciones ejecutivas según el criterio de religión.
En estas no se encontraron diferencias significativas en ninguna de las partes; por lo tanto, podemos referir que no existe una diferencia en cuanto la religión que uno profese con respecto a la toma de decisiones y las funciones ejecutivas.

Tabla 2. Comparación de medias entre grupos

\begin{tabular}{|lccccccc|}
\hline & Católico & Cristiano & Otros & Ninguno & \multicolumn{2}{c|}{ ANOVA } \\
& Media y DE & Media y DE & Media y DE & Media y DE & gl & F & Sig. \\
\hline $\begin{array}{l}\text { Total de } \\
\text { Error }\end{array}$ & $5.36 ; \pm 8.49$ & $3.5 ; \pm 5.63$ & $16 ; \pm 21.21$ & $3.79 ; \pm 7.08$ & 3 & 1.52 & 0.21 \\
$\begin{array}{l}\text { Mean } \\
\text { Accuracy }\end{array}$ & $0.84 ; \pm 0.17$ & $0.90 ; \pm 0.09$ & $0.84 ; \pm 0.14$ & $0.88 ; \pm 0.09$ & 3 & 0.6 & 0.62 \\
$\begin{array}{l}\text { Mean } \\
\text { Response }\end{array}$ & $463.29 ; \pm 85.48$ & $397.78 ; \pm 34.78$ & $483.79 ; \pm 35.67$ & $424.18 ; \pm 73.87$ & 3 & 2.28 & 0.09 \\
Time & & & & & & & \\
$\begin{array}{l}\text { Conflict } \\
\text { Cost }\end{array}$ & $53.81 ; \pm 71.74$ & $5.48 ; \pm 59.38$ & $14.27 ; \pm 6.68$ & $38.11 ; \pm 50.31$ & 3 & 1.44 & 0.23 \\
Bloque 1 & $0.31 ; \pm 5.19$ & $-1.5 ; \pm 6.02$ & $-1.00 ; \pm 1.41$ & $-0.57 ; \pm 4.86$ & 3 & 0.36 & 0.78 \\
Bloque 2 & $3.07 ; \pm 5.57$ & $5.50 ; \pm 3.82$ & $3.00 \pm 1.41$ & $3.57 ; \pm 5.50$ & 3 & 0.49 & 0.69 \\
Bloque 3 & $3.86 ; \pm 7.17$ & $4.00 ; \pm 6.76$ & $4.00 \pm 2.83$ & $5.43 ; \pm 6.63$ & 3 & 0.19 & 0.9 \\
Bloque 4 & $5.69 ; \pm 7.47$ & $5.75 ; \pm 7.13$ & $2.00 \pm 2.83$ & $4.29 ; \pm 8.30$ & 3 & 0.27 & 0.85 \\
Bloque 5 & $6.72 ; \pm 6.74$ & $2.00 ; \pm 6.14$ & $6.00 \pm 0.00$ & $4.86 ; \pm 9,07$ & 3 & 1.17 & 0.33 \\
\hline
\end{tabular}

En base al análisis post hoc, con el estadístico Tukey, no se observa influencia de la religión sobre la variable de toma de decisiones. En cuanto al análisis post hoc, con el estadístico Tukey, no se observan influencia de la religión sobre la variable de funciones ejecutivas.

\section{Discusión}

El objetivo de la investigación fue valorar si existen diferencias significativas en función de las creencias religiosas, sobre los puntajes de la toma de decisiones y el funcionamiento ejecutivo en una muestra de estudiantes universitarios. Este tema se enmarca dentro del campo de la neuropsicología cognitiva (Tirapu-Ustárroz, García, Ríos-Lagos, \& Ardila, 2012), y que corresponde a la línea de investigación del funcionamiento ejecutivo, que ha sido desarrollado en nuestra ciudad a través de diversos estudios (Denegri, \& Zegarra, 2013; Soto, \& Rondán, 2013; Zegarra, \& Chino, 2016).

En ese sentido, nuestro estudio está orientado por las teorías que plantean que en la mayoría de casos, nuestras creencias religiosas determinan nuestro patrón de 
pensamiento, y que estas se adquieren a través de la educación dada en la familia o los centros educativos (Arias, 2015), además de las convicciones personales dirigidas a alguna creencia específica (Hogan, 1973). Sin embargo, en algunos estudios locales se ha podido evidenciar que la religión no tiene un efecto diferenciador en las personas (Cotrina y Cahuata, 2017), mientras que en otros sí (Murillo, 2015). Además, se debe considerar que existen grandes diferencias en el pensamiento y los procesos de toma de decisiones, subyacentes en función de la edad (Arias y Llerena, 2013; Cotrina y Cahuata, 2017; Zegarra y Chino, 2016).

Pues bien, según nuestros resultados, no se encontró ninguna diferencia significativa entre las variables toma de decisiones y control ejecutivo, en función de la variable religiosidad que fue asumida como criterio de comparación. Podríamos atribuir estos resultados a que la muestra del presente estudio fue intencional, así como al rango de edades utilizado, puesto que se dilucida una clara influencia en cuanto a la maduración cerebral y la eficiencia del procesamiento cognitivo que esto implica.

Es decir, ya que la mayoría de estudiantes se ubican en la misma franja etaria, es posible que no se registren diferencias notorias en su forma de pensar y las creencias que asumen. Pero también podría deberse a que las creencias religiosas de los estudiantes no son asumidas de forma consistente con su práctica, a pesar de que se consideró el criterio de comparación, entre creyentes practicantes y no practicantes, para quienes indicaron que tenían alguna religión como base de sus creencias. En ese sentido, sería necesario que para futuras investigaciones, se trabaje con un muestreo probabilístico y con grupos equivalentes, para llegar a resultados más concluyentes (Castro, Arias, Dominguez, Masías, Salas, Canales y Flores, 2013). 


\section{Referencias}

Arias, W. L. (2015). Estudio de los valores morales en el Perú: una propuesta para la Educación. Educationis Momentum, 1(1), 33-59.

Arias, W. L., \& Llerena, S. (2013). Prosocialidad en niños de edad pre-escolar de la Institución Educativa Santísimo Salvador. Véritas, 14(1), 11-16.

Ato, M., López, J. J., \& Benavente, A. (2013). Un sistema de clasificación de los diseños de investigación en psicología. Anales de Psicología, 29(3), 1038-59.

Baumnister, R. F., Bauer, I. M., \& Lloyd, S. A. (2010). Choice, free will and religion. Psychology of Religion and Spirituality, 2(2), 67-82.

Baumeister, R. F., \& Exline, J. J. (1999). Virtue, Personality, and social relations: Selfcontrol as the moral muscle. Journal of Personality, 67(6), 1165-1194.

Bechara, A., Damasio, A., Damasio, H., \& Anderson, S. (1994). Insensitivity to future consequences following damage to human prefrontal cortex. Cognition, 50, 7-12.

Bechara, A., Damasio, H., Tranel, D., \& Damasio A. (2005). The Iowa Gambling Task and the somatic marker hypothesis: some questions and answers. Trends in Cognitive Science, 9, 159-162.

Castro, R., Arias, W., Dominguez, S., Masías, M. A., Canales, F., \& Flores, A. (2013). Integración familiar y variables socioeconómicas en Arequipa metropolitana. Revista de Investigación, 4, 35-65.

Cotrina, M., \& Cahuata, B. (2017). Desarrollo del juicio moral según el plan de estudios de dos universidades de Arequipa. Revista de Psicología (Universidad Católica San Pablo), 7(1), 33-47.

Denegri, L., \& Zegarra, J. (2013). Funcionamiento cognitivo y cognición social en el trastorno obsesivo-compulsivo. A propósito de un caso. Revista de Psicología de Arequipa, 3(1), 79-88.

Eriksen, B. A., \& Eriksen, C. W. (1974). Effects of noise letters upon identification of a target letter in a non- search task. Perception and Psychophysics, 16, 143-149. 
Gordillo, F., Arana, J., Salvador, J., \& Hernández, M. (2011). Emoción toma de decisiones: teoría y aplicación del IOWA GAMBLING TASK. Revista Electrónica de Psicología Iztacala, 14(1), 333-353.

Hernández, R., Fernández, C., \& Baptista, P. (2006). Metodología de la investigación. México: McGraw-Hill.

Hogan, R. (1973). Moral Conduct and moral caracter: A psychological perspective. Psychological Bulletin, 79, 217-232

Murillo, M. G. (2015). Desarrollo del juicio moral en personas casadas y divorciadas de la ciudad de Arequipa. Avances en Psicología, 23(1), 73-85.

Osorio, J. F. (2011). Competencia del juicio moral en escolares de quinto año de secundaria y universitarios de primer semestre en la ciudad de Arequipa. Revista de Psicología (Universidad Católica San Pablo), 1(1), 28-38.

Papalia, D., Wendkos, S., \& Duskin, R. (2009). Desarrollo humano. México D. F.: McGraw-Hill.

Soto, M., \& Rondán, D. (2013). Funciones ejecutivas en pacientes con esquizofrenia. Revista de Psicología de Arequipa, 3(2), 143-152.

Stelzer, F., Cervigni, M., \& Martino, P. (2010). Bases neurales del desarrollo de las funciones ejecutivas durante la infancia y adolescencia. Una revisión. Revista Chilena de Neuropsicología, 5(3), 176-184.

Tiplady, B., Degia, A., \& Dixon, P. (2005). Assessment of driver impairment: Evaluation of a two-choice tester using ethanol. Traffic Psychology and Behavior, 8, 299-310.

Tirapu-Ustárroz, J., García, A. Ríos-Lagos, M., \& Ardila, A. (2012). Neuropsicología de la corteza prefrontal y de las funciones ejecutivas. Barcelona: Viguera.

Zegarra, J. A., \& Chino, B. N. (2016). Impacto de la religión en la toma de decisiones y el funcionamiento ejecutivo. Revista de Psicología (Universidad Católica San Pablo), 6(2), 59-73.

Recibido: 7 de noviembre de 2019

Aceptado: 7 de agosto de 2020 